This is a post-print author self-achieve version. The paper has been published on AICHE Journal: DOI 10.1002/aic.14837

\title{
Accuracy and Optimal Sampling in Monte Carlo Solution of Population Balance Equations
}

Xi Yu ${ }^{1,2, *}$, Michael J Hounslow ${ }^{2}$, Gavin K Reynolds ${ }^{3}$

1 European Bioenergy Research Institute (EBRI), School of Engineering and Applied Science, Aston University, Birmingham B4 7ET, UK

2 Department of Chemical and Biological Engineering, the University of Sheffield, Sheffield, S1 3JD, UK

3 Pharmaceutical Development, AstraZeneca, Macclesfield, SK10 2NA, UK

*Corresponding concerning this article should be addressed to Xi Yu at x.yu3@aston.ac.uk

\section{Abstract}

Implementation of a Monte Carlo simulation for the solution of population balance equations requires choice of initial sample number $\left(N_{0}\right)$, number of replicates $(M)$ and number of bins for probability distribution reconstruction (n). It is found that Squared Hellinger Distance, $\mathrm{H}^{2}$, is a useful measurement of the accuracy of $M C$ simulation, and can be related directly to $N_{0}, M$ and $n$. Asymptotic approximations of $H^{2}$ are deduced and tested for both $1 D$ and $2 D$ PBEs with coalescence. The $C P U$ cost, $C$, is found in a power-law relationship, $C=$ $a M N_{0}^{b}$, with the CPU cost index, $b$, indicating the weighting of $N_{0}$ in the total CPU cost. $n$ must be chosen to balance accuracy and resolution. For fixed $n$, $M \times N_{0}$ determines the accuracy of $M C$ prediction; if $b>1$, then the optimal solution strategy uses multiple replications and small sample size. Conversely if $0<b<1$, one replicate and a large initial sample size is preferred.

Keywords: Monte Carlo, Population Balance Model, Hellinger Distance, Optimal Sampling, Accuracy, Coalescence

\section{Introduction}


Population balance equations (PBEs) describe the evolution of the properties of a collection of particles (eg crystals, agglomerates, soot) in time and perhaps space ${ }^{1-3}$. Such equations usually require numerical solution frequently via a stochastic technique. Monte Carlo simulation (MCS) has been used as such a method over the past few decades ${ }^{4,5}$. With this approach a large population of particles, perhaps of $\mathrm{O}\left(10^{9}\right)$ is represented by a small sample, perhaps $\mathrm{O}\left(10^{3}\right)$. Each particle is then simulated by evolving its properties (or internal coordinates, such as size or composition), via mechanisms that involve interaction between particles, selected in some random way, hence the analogy with Monte Carlo methods.

For a coalescence phenomenon in a closed system, as described in this paper, the number of particles will decline over time. Therefore, if the size of the simulation box (ie the apparent size of the space represented by the sample particles) is kept constant, the number of particles used to represent the real system will also decline. Two event-driven methods, stepwise constant volume Monte Carlo (SCVMC) $)^{6,7}$ and constant number Monte Carlo $(\mathrm{CNMC})^{8}$ have been devised to circumvent this problem and are widely used to solve PBEs. In the SCVMC method, the volume is halved or doubled when the particle number increases or decreases by a factor of two of its initial value, respectively. In the CNMC approach, the volume is continuously adjusted to keep number of particle constant in the virtual simulation box. Maisels et $\mathrm{al}^{7}$ demonstrated the prediction of the SCVMC method is more accurate than the CNMC method for nucleation and coagulation problems.

\section{The choice of $N_{0}, M$ and $n$}

The principle of the stochastic MC method for solution of PBEs is that the dynamic evolution of an extremely large population of particles can be represented by monitoring the corresponding discrete events occurring in a smaller number of sample particles. Therefore, sampling a finite number of particles appropriately is crucial to describing population dynamics and prediction of product quality in real systems. Three essential parameters, including initial sample number $\left(N_{0}\right)$, number of replicates $(M)$ and number of size bins $(n)$, need to be chosen if we run an MC simulation and compare with theoretical or experimental data. The number of replicates $(M)$ in this study indicates how many times an $M C$ simulation needs to be run. A review of the published sampling strategies using the MC method from a 
range of applications is shown in Table 1 , the scope of this survey covers $N_{0}, M$, CPU cost and goodness of fit. Almost all the MC applications examine $10^{3}-10^{6}$ particles at a time on PCs of different ages because of the limitation of CPU speed and memory capacity. Fewer studies consider the computation time of the MC algorithm for some specific application on PCs of different ages. Some researchers ${ }^{13}$, 17 comment qualitatively that the accuracy of MC (the relative error between predicted and theoretical values) is proportional to $1 / \sqrt{N}$ where $N$ is the number of particles in the system. Smith and Matsoukas ${ }^{4}$ quantitatively proved the correlation between MC error, $\delta$ and $1 / \sqrt{N}$ by fitting simulation results. A successful representative population in accuracy-constrained MC simulation can be achieved in two ways: by running the MC program once with a very large initial sample number, or by combining the results of several runs, each with a smaller sample number ${ }^{14}$. However, none of previous publications specify how to choose $N_{0}, M$ and $n$ to achieve a specific accuracy with acceptable computational cost. In this study, we aim to

I. use the Hellinger Distance, a statistical distance between two probability distributions, to measure the accuracy of Monte Carlo simulations.

II. provide guidance on how to select the initial sample number and number of replicates and consider tradeoffs between accuracy and computational costs. 
Table 1: Summary of application of Monte Carlo method to PBEs in the literatures

\begin{tabular}{|c|c|c|c|}
\hline Application (Ref. and Authors) & $N_{0}(M)$ & Computational time & Goodness of fit \\
\hline Bipolar charging ${ }^{7}$ & - & - & - \\
\hline Coalescence in a cloud $d^{10,11}$ & - & - & - \\
\hline Coupled chemical reaction ${ }^{12}$ & - & - & - \\
\hline $\begin{array}{l}\text { chemical reaction } \\
\text { and coating }\end{array}$ & $\begin{array}{l}3 \times 10^{4}(4) \\
2 \times 10^{4}(5)\end{array}$ & - & Qualitative comment on accuracy via $1 / \sqrt{N}$ \\
\hline Crystallization $^{14}$ & $6400-25600(1-250)$ & 3-620mins in PCs & $\begin{array}{c}\text { Good statistical representative is achieved in two } \\
\text { ways }\end{array}$ \\
\hline Fractal aggregation ${ }^{15}$ & $3-100(1000)$ & - & - \\
\hline Higher dimensionality problems ${ }^{16}$ & - & - & - \\
\hline Multi-component aerosols ${ }^{17}$ & $3 \times 10^{6}(10)$ & $\begin{array}{l}\text { A few hours(256Mb RAM and } \\
\text { Pentium III 900-Mhz pc) }\end{array}$ & Qualitative comment on accuracy via $1 / \sqrt{N}$ \\
\hline $\begin{array}{l}\text { Particle Aggregation and } \\
\text { restructuring }\end{array}$ & $\begin{array}{c}10^{4}(15) \\
10^{4}(100)\end{array}$ & $\begin{array}{c}\text { 2h (SPRAC -ten workstation) } \\
--\end{array}$ & $\begin{array}{l}\text { Average/ensemble method } \\
\quad \text { Average procedure }\end{array}$ \\
\hline Wet scavenging ${ }^{19}$ & 3000 & $25-78 \mathrm{~s}$ in $\mathrm{PCs}$ & - \\
\hline Wet Granulation ${ }^{20-25}$ & $1024-4096(64)$ & - & - \\
\hline
\end{tabular}




\section{Theory}

\section{Squared Hellinger Distance}

The Hellinger Distance is used to quantify the similarity between two probability distributions. The most ubiquitous application of Hellinger distance is minimum Hellinger distance estimation ${ }^{26-29}$ in statistics.

Squared Hellinger Distance $\left(H^{2}\right)$ between distribution function $f$ and $g$ is

$$
H^{2}(f, g)=\frac{1}{2} \int(\sqrt{f(x)}-\sqrt{g(x)})^{2} d x
$$

where $f(x), g(x)$ are probability density functions (PDF), describing the frequency of occurrence at size $x(1 / \mathrm{m})$, where $x$ is particle size $(\mathrm{m})$. If $f$ and $g$ are identical, $H^{2}=$ 0 ; if the two distributions do not overlap at all, $H^{2}=1$. In this way $H^{2}$ provides a scaled, dimensionless measurement of accuracy that ranges between 0 and 1 .

The PDFs in Eq. 1 are both continuous and normalized (i.e. have a zeroth moment of 1) whereas the results from MC simulation are discrete and not normalized. An appropriate modification to Eq. 1 to allow for comparison of discrete MC results with continuous analytical results is:

$$
\begin{gathered}
H^{2}\left(f_{M C}, f_{\mathrm{AS}}\right)=\frac{1}{2} \sum_{i}\left(\sqrt{\frac{N_{i}}{\sum_{C_{i}} N_{i}}}-\sqrt{\frac{\int_{C_{i}} n(\vec{x}, t) d \vec{x}}{\int_{\forall \vec{x}} n(\vec{x}, t) d \vec{x}}}\right)^{2} \\
f_{M C}(\vec{x})=\frac{N_{i}}{\Delta \vec{x}_{i} \sum_{C_{i}} N_{i}}, f_{\mathrm{AS}}(\vec{x})=\frac{n(\vec{x}, t)}{\iint_{\forall \vec{x}} n(\vec{x}, t) d \vec{x}}
\end{gathered}
$$

Where $N_{i}$ is number in size bin $C_{i}$ in the MC simulation, subscript AS refers to the analytical solution.

\section{Coalescence PBEs}

In this study, two cases, 1D size-dependent and 2D size-independent coalescence PBEs with analytical solutions are examined. These cases are selected as they present significant differences in algorithm structure and evolving distribution of particle properties, which make a major impact on the correlations of computational time and accuracy with $N_{0}$, and $M$ in MCS.

Case1: 1D size-dependent 
Gelbard and Seinfeld ${ }^{30}$ produced a result for the coalescence of an exponential distribution with a kernel given by $\beta\left(m_{1}, m_{2}\right)=\beta_{0}\left(m_{1}+m_{2}\right)$. Here, $m_{1}$ and $m_{2}$ are the mass of colliding particles. The initial distribution function of particles volume is

$$
n(m, 0)=\frac{n_{0}}{m_{0}} \operatorname{Exp}\left(-\frac{m}{m_{0}}\right)
$$

The analytical solution for population density function at time $t$ is

$$
\begin{gathered}
n(m, t)=\frac{n_{0}(1-T(t))}{m \sqrt{T(t)}} \operatorname{Exp}\left(-(1-T(t)) \frac{m}{m_{0}}\right) I_{1}\left(2 \frac{m}{m_{0}} \sqrt{T(t)}\right) \\
T(t)=1-\operatorname{Exp}\left(-n_{0} b_{0} m_{0} t\right)
\end{gathered}
$$

Where $I_{1}$ is Bessel function of the first kind of order one. $n(m, 0), n(m, t)$ are number density functions (NDF) at time 0 and $t$, respectively.

\section{Case2: 2D size-independent}

A two dimensional analytical solution for the size independent coalescence PBE is revealed by Vale and McKenna ${ }^{31}$, in view of solution proposed by Gelbard and Seinfeld ${ }^{30}$. The initial distribution of particles which have two components in mass mode is

$$
n\left(m_{1}, m_{2}, 0\right)=\frac{16 n_{0}}{m_{10} m_{20}}\left(\frac{m_{1}}{m_{10}}\right)\left(\frac{m_{2}}{m_{20}}\right) \operatorname{Exp}\left(-2 \frac{m_{1}}{m_{10}}-2 \frac{m_{2}}{m_{20}}\right)
$$

Where $n_{0}$ is initial number of particles per unit volume; $m_{i 0}$ is initial mean mass of the $i^{\text {th }}$ component in a particle.

For a constant coalescence coefficient $\beta_{0}$, the analytical solution is

$$
\begin{gathered}
n\left(m_{1}, m_{2}, \tau\right)=\frac{8 n_{0}}{m_{10} m_{20} \sqrt{\tau(\tau+2)^{3}}} \operatorname{Exp}\left(-2 \frac{m_{1}}{m_{10}}-2 \frac{m_{2}}{m_{20}}\right)\left(I_{0}(\theta)-J_{0}(\theta)\right) \\
\text { where } \theta=4\left(\frac{m_{1} m_{2}}{m_{10} m_{20}}\right)^{1 / 2}\left(\frac{\tau}{\tau+2}\right)^{1 / 2} \\
\tau=n_{0} \beta_{0} t
\end{gathered}
$$

Where, $J_{0}(\theta)$ is Bessel function of the first kind; $I_{0}(\theta)$ is Modified Bessel function of the first kind.

Table 2 lists the parameters used in the MC simulation. 
Table 2: Parameters used in MC simulation

\begin{tabular}{cccc}
\hline \multicolumn{2}{c}{ 1D Size dependent } & \multicolumn{2}{c}{ 2D Size independent } \\
parameter & Value & parameter & value \\
\hline$b_{0}$ & 1 & $B_{0}$ & 1 \\
$n_{0}$ & 1 & $n_{0}$ & 1 \\
$b_{0}$ & 1 & $m_{10}$ & 1 \\
$m_{0}$ & 1 & $m_{20}$ & 5 \\
$t$ & 6 & $\tau$ & 100 \\
$I_{\text {agg }}$ & 0.99 & $I_{\text {agg }}$ & 0.99 \\
$N_{0}$ & $50-5,000$ & $N_{0}$ & $500-50,000$ \\
$M$ & $1-20$ & $M$ & $1-20$ \\
$n$ & $13-13,000$ & $n$ & $7^{2}-350^{2}$ \\
\hline
\end{tabular}

\section{Asymptotic approximation of $\mathrm{H}^{2}$}

We consider now two asymptotic cases: that where the number of particles in all size ranges is large, and that when it is small. The expected value of a discrete approximation to the $H^{2}$ is based on the following assumptions: (1) the number of particles, $\widehat{N}_{k}$, in each size range $C_{k}$ in MC results has a Poisson distribution; (2) A group of $\widehat{N}_{k}$ 's are uncorrelated amongst themselves over the domain.

To obtain the expected value of a discrete approximation to the $H^{2}$. Eq. 2 is written as,

$$
H^{2}=\frac{1}{2 M N_{0}} \sum_{k=1}^{n}\left(\sqrt{N_{k}}-\sqrt{\widehat{N}_{k}}\right)^{2}
$$

Where $N_{k}$ is the number associated with $k^{\text {th }}$ internal and $\widehat{N}_{k}$ is an estimate of that number and

$$
M N_{0}=\sum_{k=1}^{n} N_{k}=\sum_{k=1}^{n} \widehat{N}_{k}
$$

If we assume that the values of $\widehat{N}_{k}$ have a Poisson distribution of $N_{k}$, i.e. the probability that $\widehat{N}_{k}$ takes on a value $x$ is

$$
P_{\widehat{N}_{k}}(x)=\frac{e^{-N_{k}} N_{k}^{x}}{x !}
$$

Then the expected value of $H^{2}$ is

$$
E\left[H^{2}\right]=\frac{1}{2 M N_{0}} E\left[\sum_{k=1}^{n}\left(\sqrt{N_{k}}-\sqrt{\widehat{N}_{k}}\right)^{2}\right]=\frac{1}{2 M N_{0}} \sum_{k=1}^{n}\left(N_{k}-2 \sqrt{N_{k}} E\left[\sqrt{\widehat{N}_{k}}\right]+E\left[\widehat{N}_{k}\right]\right)
$$




$$
=\frac{1}{N_{0}} \sum_{k=1}^{n}\left(N_{k}-\sqrt{N_{k}} E\left[\sqrt{\widehat{N}_{k}}\right]\right)
$$

However if $N_{k}$ is everywhere small $E\left[\widehat{\widehat{N}}_{k}\right]=N_{k}$ so

$$
E\left[H^{2}\right]=\frac{1}{N_{0}} \sum_{k=1}^{n}\left(N_{k}-N_{k} \sqrt{N_{k}}\right)=\frac{1}{M N_{0}} \sum_{k=1}^{n}\left(N_{k}-N_{k} \sqrt{N_{k}}\right)=1-\frac{1}{M N_{0}} \sum_{k=1}^{n} N_{k} \sqrt{N_{k}}
$$

Now, the $N_{k}$ scale with $M N_{0} / n$ so put $M N_{0} N_{k}^{1} / n$ where the $N_{k}^{1}$ are constants. Therefore

$$
E\left[H^{2}\right]=1-\left(\frac{M N_{0}}{n}\right)^{0.5} \sum_{k=1}^{n} N_{k}^{1} \sqrt{N_{k}^{1}}=1-a^{\prime}\left(\frac{M N_{0}}{n}\right)^{0.5}
$$

Where $a^{\prime}$ is a constant independent of $M N_{0}$ and $n$.

If $N_{k}$ is everywhere large, the expected value of $\sqrt{\widehat{N}_{k}}$ is given by Kendall and Stuart ${ }^{32}$,

$$
E\left[\sqrt{\widehat{N}_{k}}\right]=\sqrt{N_{k}}-\frac{1}{8} N_{k}^{-1 / 2}-o\left(N_{k}^{-3 / 2}\right)
$$

Combining Eq. 14 and 17

$$
E\left[H^{2}\right]=\frac{1}{N_{0}} \sum_{k=1}^{n}\left(\frac{1}{8}+o\left(N_{k}^{-1}\right)\right) \cong \frac{n}{8 M N_{0}}=\frac{1}{8}\left(\left(\frac{M N_{0}}{n}\right)^{1 / 2}\right)^{-2}
$$

The expected value of a discrete approximation to the $H^{2}$ in Eq. 16 and 18 shows the relationship of $H^{2}$ with $\left(M N_{\delta} / n\right)^{1 / 2}$. This suggests a plot of $H^{2}$ against $\left(M N_{0} / n\right)^{1 / 2}$.

\section{Simulation methods}

A flowchart of MC solution of coalescence PBEs is shown in in Fig.1. The particle population is represented in an array with $N_{p}$ rows to represent each individual particle and $N_{i}$ columns for each internal coordinate. The shorthand $X_{j, k}$ is used to refer to the $k^{\text {th }}$ internal coordinate of $j^{\text {th }}$ the particle (row $j$ column $k$ in the array). To represent the initial particle population, each cell in the array is initialized using the generation procedure, transformation $\operatorname{method}^{9}$ in one dimensional PBEs and conditional distribution method ${ }^{9}$ in two dimensional PBEs.

With the array (Fig.1) initialized, the coalescence rate can be estimated to control the property evolution of the particle population at each time step. The time interval is calculated from the coalescence rate, so that there is a coalescence event per time interval. The time interval is 


$$
\Delta t=\frac{1}{r_{\text {coal }}}
$$

The rate for size-dependent coalescence is calculated from a coalescence table. Each cell of coalescence table represents the value of a coalescence kernel e.g. for $\beta\left(v_{l}, v_{m}\right)=v_{l}+v_{m}$, the average coalescence kernel is ${ }^{4}$

$$
\bar{\beta}\left(v_{l}, v_{m}\right)=\frac{2}{N_{P}\left(N_{P}-1\right)} \sum_{m=l+1}^{N_{P}} \sum_{l=1}^{N_{P}-1} \beta\left(v_{l}, v_{m}\right)
$$

The rate for size-dependent coalescence is

$$
\begin{gathered}
r_{\text {coal }}=\frac{1}{2} \bar{\beta}\left(v_{l}, v_{m}\right) V C_{n}^{2} \\
C_{n}=\frac{N_{P}}{V}
\end{gathered}
$$

The rate for size-independent coalescence is

$$
r_{\text {coal }}=\frac{1}{2} \beta_{0} V C_{n}^{2}
$$

Where $\beta_{0}$ is the coalescence rate constant, $V$ is the sample volume in the MC simulation, $C_{n}$ is total particle number per unit volume in the physical system.

In this algorithm, assumed array size (AAS) and dynamic allocation of array (DAA) are used to store and update the properties of particle population over time. The AAS approach declares an array with a fixed size. The DAA approach dynamically allocates an array of the right size or reallocates an array when it needs to expand.

\section{AAS}

In the CNMC algorithm, the array is updated in the case of a coalescence event in three steps (Fig 1):

(1) Replace the property information of particle $m_{j 1}$ (row $j 1$ of the array) with $m_{j 1}+m_{j 2}$.

(2) Randomly select particle $m_{j 3}$ (row $j 3$ of the array). ( $j 3 \neq j 1$ or $j 2$ )

(3) Replace the property information of particle $m_{j 2}$ (row j2 of the array) with $m_{j 3}$ In the SCVMC algorithm, the array is updated for a coalescence event in three steps:

(1) Replace the property information of particle $m_{j 1}$ (row $j 1$ of the array) with $m_{j 1}+m_{j 2}$.

(2) Replace the property information of particle $m_{j 2}$ row $j 2$ of the array) with $m_{j N}$

(3) Set property information of particle $m_{j N}=0$ ( row $j N$ of the array)

\section{$D A A$}

In the SCVMC algorithm, the array is updated for a coalescence event in two steps (Fig 1): 
(1) Replace the property information of particle $m_{j 1}$ (row $j 1$ of the array) with $m_{j 1}+m_{j 2}$.

(2) Remove the property information of particle $m_{j 2}$ (row $j 2$ of the array)

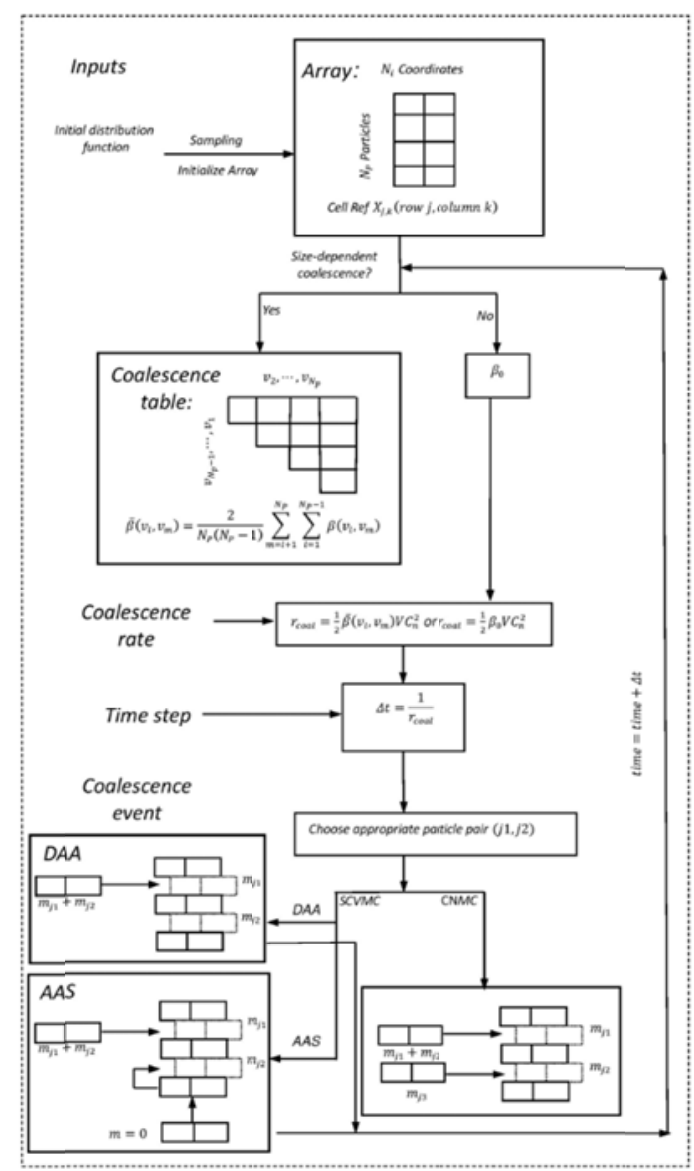

Fig 1. Flowchart of MC solution of coalescence PBEs

\section{Results and discussion}

\section{Case1: 1D size-dependent}

The accuracy of MC simulation is closely related to $N_{0}$ and $M$. The impact of $N_{0}$ and $M$ on accuracy of the MC results for 1D PBEs with size-dependent coalescence were examined in Fig. 2. The predictive distributions of the CNMC approach are compared to the analytical solution (Eq.5) at a case with $I_{a g g}=0.99 .^{33}$ In Fig. 2a, some data points are scattered randomly and deviated from the theoretical curve at small $N_{0}$ $(<500)$. $H^{2}$ declines from 0.21 to 0.004 as $N_{0}$ increased from 50 to 5,000 . As $M$ increases from 1 to 20 in Fig. 2b, both the decreases of degree of scatter and $H^{2}$, showing a significant increase in the accuracy. Fig.2c shows the consistent accuracy of $\mathrm{MC}$ simulation when $M \times N_{0}$ is kept constant. 

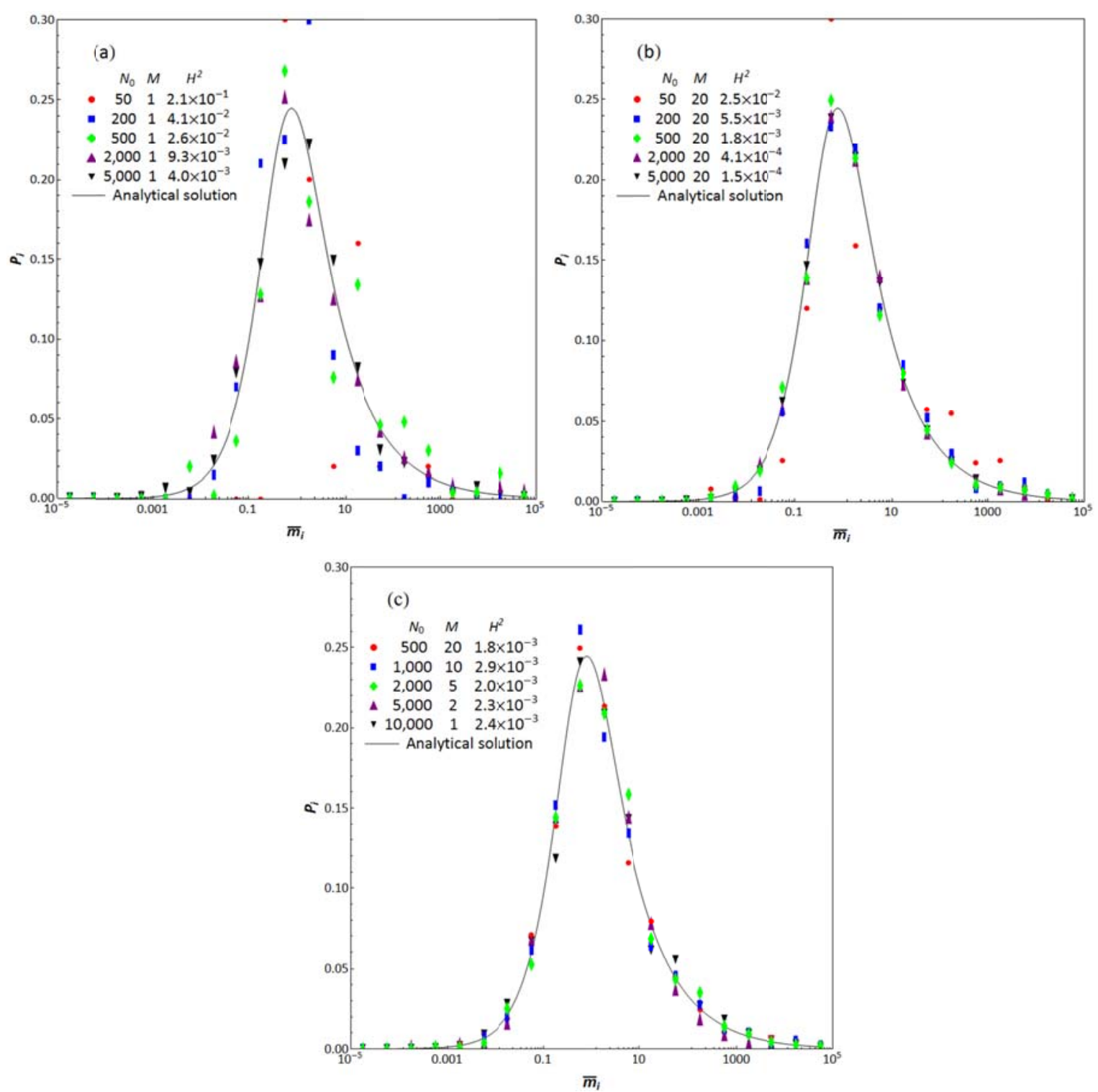

Fig 2. Comparison between particle mass distribution (at constant size bins $n=21$ ) obtained by CNMC $\left(P_{i}=P\left(m_{i} \leq m<m_{i+1}\right), \bar{m}_{i}=\left(m_{i+1}+m_{i}\right) / 2\right)$ and analytical solution $\left(P_{i}\left(\bar{m}_{i}\right)=\int_{m_{i}}^{m_{i+1}} n(m) d m\right)$ for $1 \mathrm{D}$ case (size-dependent coalescence) (a) at constant $M=1$ (b) at constant $M=20$ (c) at constant $M N_{0}=10,000$ 


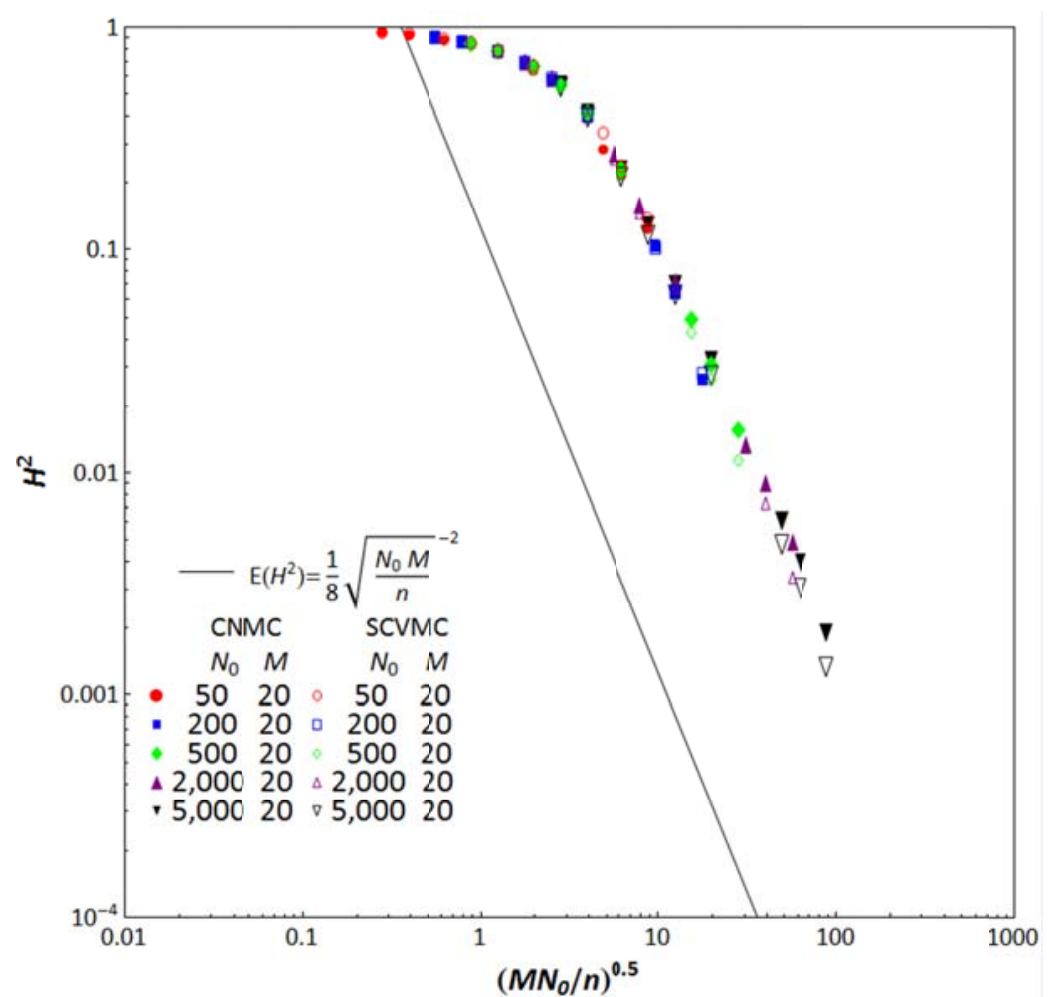

Fig 3.Calculated (CNMC and SCVMC at size bins $n=13-13,000$ ) and asymptotic approximation $H^{2}$ (when $\left(M N_{0} / n\right)^{1 / 2}$ is large) dependence of $\left(M N_{0} / n\right)^{1 / 2}$ for $1 \mathrm{D}$ case (size-dependent coalescence) at constant $M=20$

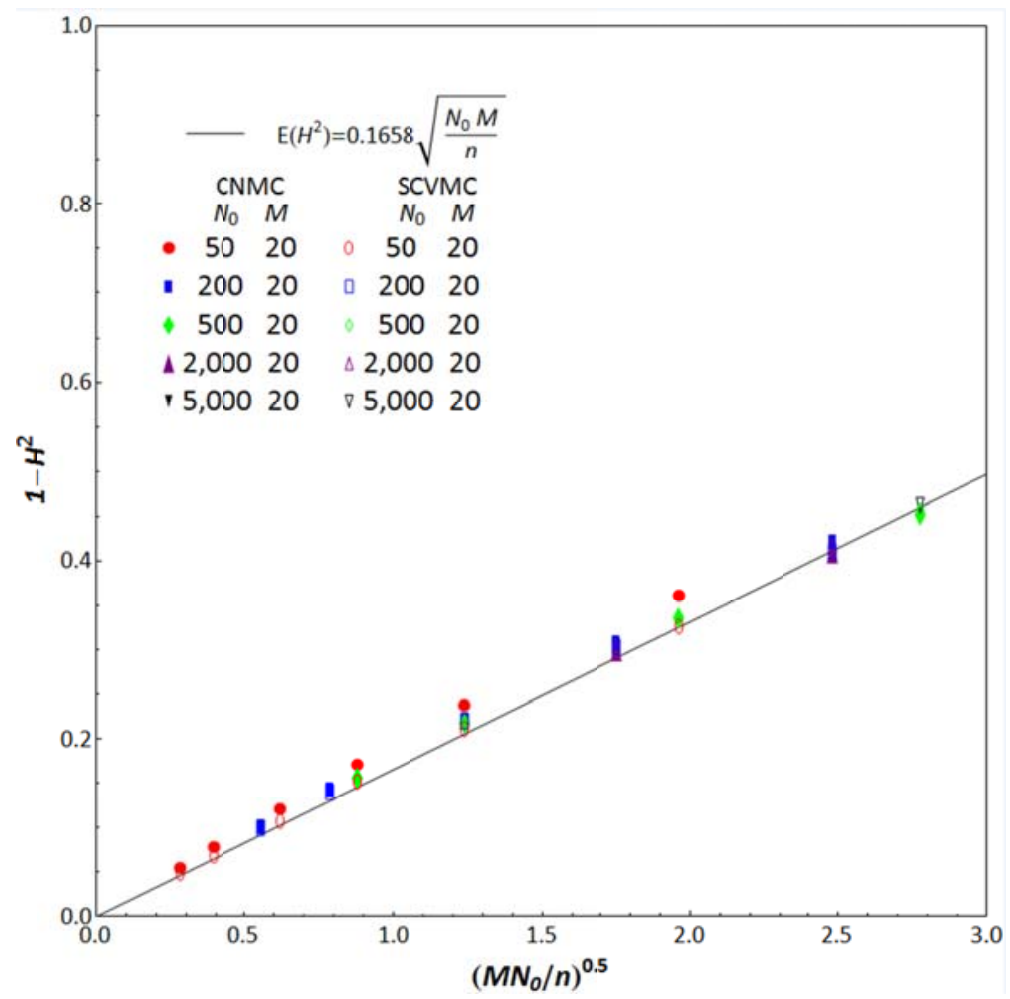

Fig 4. Calculated (CNMC and SCVMC at size bins $n=13-13,000)$ and asymptotic approximation $1-H^{2}$ (when $\left(M N_{0} / n\right)^{1 / 2}$ is small) dependence of $\left(M N_{0} / n\right)^{1 / 2}$ for $1 \mathrm{D}$ case (size-dependent coalescence) at constant $M=20$

The theoretical correlation between $H^{2}$ and $\left(M N_{0} / n\right)^{1 / 2}$ is shown in Fig 3 and 4 for the 
1D size-dependent coalescence PBE case using the CNMC and SCVMC approaches. According to Eq. 18, asymptotic approximation of $H^{2}$ is a power law of $\left(M N_{0} / n\right)^{1 / 2}$ with a slope -2 when $\left(M N_{0} / n\right)^{1 / 2}$ is large. Furthermore, the critical value of $H^{2}$ should be $1 / 8$, at $\left(M N_{d} / n\right)^{1 / 2}=1$. In Fig 3, the simulated $H^{2}$ (CNMC and SCVMC) is over-predicted compared to the asymptotic approximation curve of $H^{2}$. The reason for this can be explained by the limitation of our assumption in the derivation process of the asymptotic approximation of $H^{2}$. It is assumed that $\widehat{N}_{k}$ 's are uncorrelated among themselves over the domain and are everywhere large. This latter assumption cannot be valid when $N_{0}$ is small. However, it is noted that the observed values of $H^{2}$ do scale as expected when $\left(M N_{d} / n\right)^{1 / 2}>3$ in Fig. 3. According to Eq. 16, the expected value of $1-H^{2}$ is a linear function of $(M N d n)^{1 / 2}$ when $\left(M N_{d} / n\right)^{1 / 2}$ is small. The prediction trends from both the CNMC and SCVMC approaches are consistent with the theoretical curve when $\left(M N_{d} / n\right)^{1 / 2}<3$ in Fig 4 . However in this regime, error, or $H^{2}$, is always large and so should be avoided. It is noted that $n$ determines the resolution of MC prediction, and the accuracy of MC simulation decreases as $n$ increases. Essentially, small $n$ is always to be avoided (e.g. $n=1$ ). It is worth noting that the choice of algorithm has very little impact on accuracy.

\section{Case2: 2D size-independent}

The theoretical NDF 3D plots on the $m_{1} m_{2}$ plane for the analytical solution (Eq. 7 and 8 ) of the $2 \mathrm{D}$ size-independent coalescence PBE at $\mathrm{T}=0$ and $\mathrm{T}=100$ are shown in Fig 5. Particle number density is significantly decreased at $\mathrm{T}=100$ due to coalesecence. 

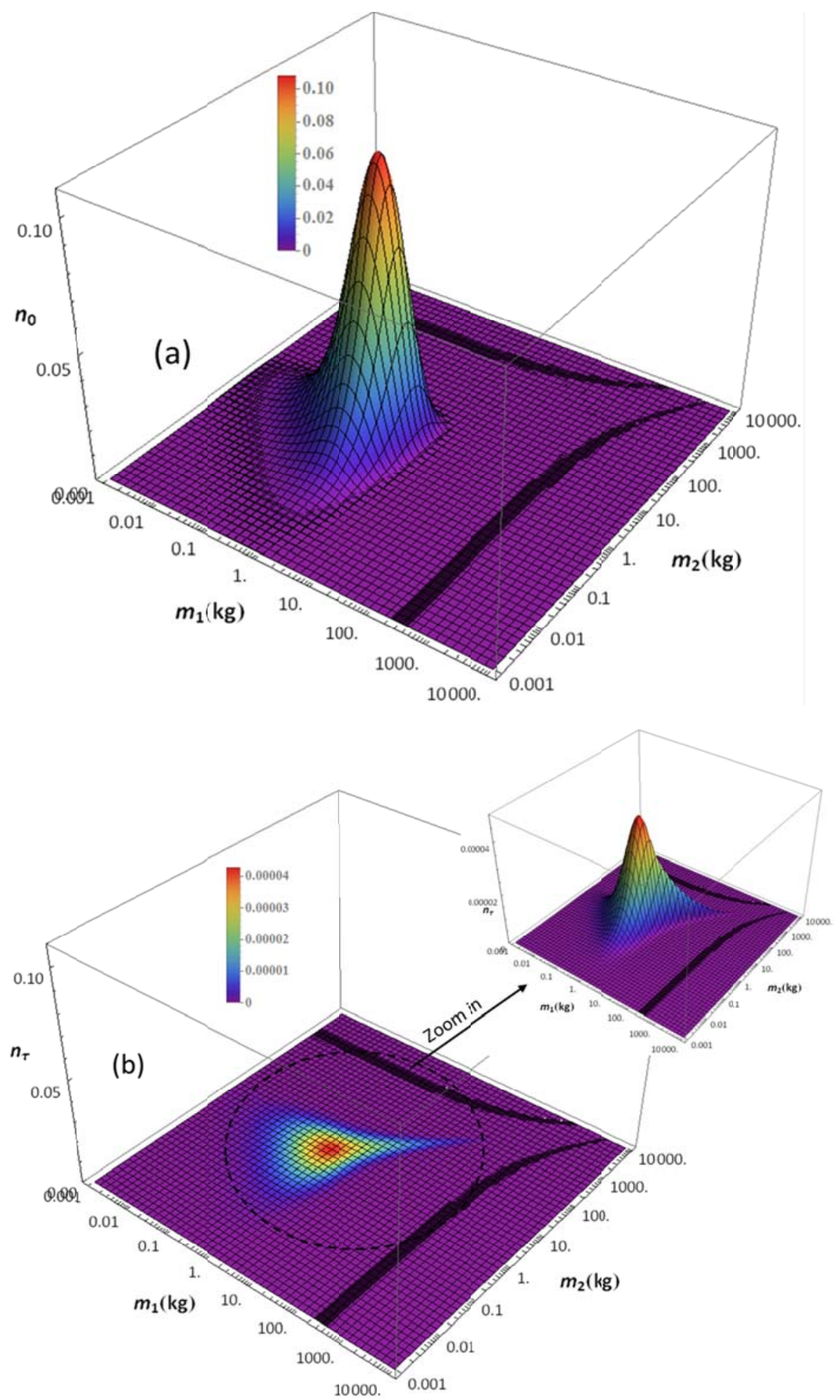

Fig 5. 3D plot of particle mass $\left(m_{1}, m_{2}\right)$ distribution of analytical solution for 2D case (sizeindependent coalescence) (a) at $\tau=0\left(n_{0, \max }=0.108\right)(\mathrm{b})$ at $\tau=100\left(n_{\tau, \max }=4.29 \times 10^{-5}\right)$ The dependence of $H^{2}$ on $\left(M N_{0} / n\right)^{1 / 2}$ is shown in Fig 6 for this case using the CNMC and SCVMC approaches. Also shown is the asymptotic prediction which is in reasonable agreement with the simulation results. There is some evidence that the CNMC algorithm outperforms SCVMC, presumably because in this case there is 
considerable reduction in simulated particle numbers.

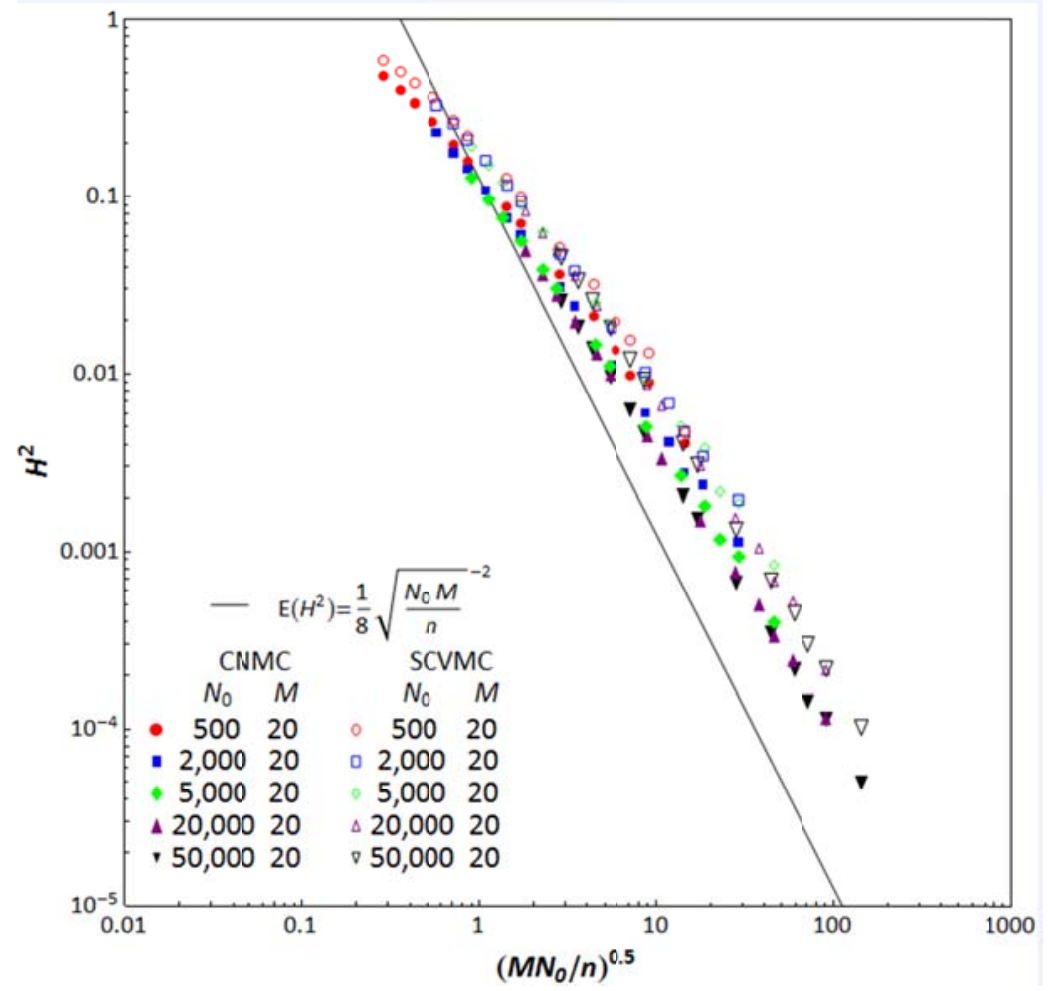

Fig 6.Calculated (CNMC and SCVMC at size bins $n=7^{2}-350^{2}$ ) and asymptotic approximation $H^{2}$ (when $\left(M N_{0} / n\right)^{1 / 2}$ is large) dependence of $\left(M N_{0} / n\right)^{1 / 2}$ for 2D case (size-independent coalescence) at constant $M=20$

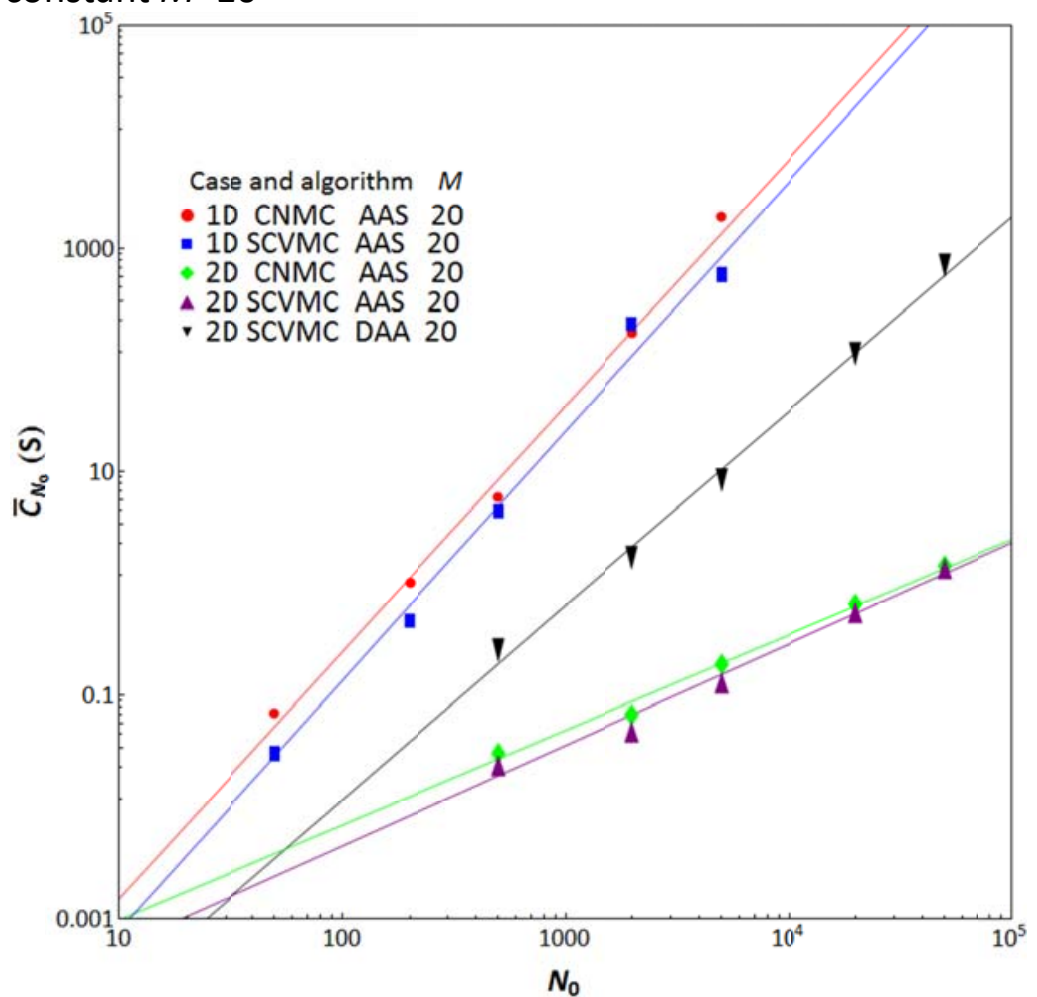

Fig 7. Averaged (constant $M=20) \mathrm{CPU}$ cost dependence of $N_{0}$ for $1 \mathrm{D}$ case (size-dependent coalescence) using methods (CNMC, SCVMC) and 2D case (size-independent coalescence) using methods (CNMC, SCVMC (AAS and DAA)), the CPU cost index (b) of each MC algorithm 
is in Table 3

\section{CPU cost}

In this section, the correlation of computational time (CPU cost) with $N_{0}$ and $M$ is examined for the assessment of computational efficiency. The computational time $C$ of a $\mathrm{MC}$ simulation is seen to follow a power law relationship in initial sample number $N_{0}$ and is a linear function of replication $M$

$$
C=a N_{0}^{b} M
$$

Where $a$ and $b$ are unknown parameters; we term $b$ the CPU cost index. A series of $M C$ simulations at different $N_{0}$ and constant $M=20$ were implemented and CPU costs recorded. The interrelationship between CPU cost and $N_{0}$ is shown in Fig 7. A linear regression approach is used to estimate $a$ and CPU cost index $b$, which are shown in Table 3. Both parameters are remarkably sensitive to the computational complexity of the algorithm. The difference in the parameters between 1D and 2D case is due to the coalescence algorithm. The algorithm of size-dependent coalescence used nested DO-Loops to build the coalescence table for calculating coalescence rate. This sub-process needs to recall storage memory in the order of $N_{0}^{2}$. In DAA, $N-1$ storage locations of the previous array with size $N-1$ need to be recalled and replicated into a new array with size $N-1$. This implementation leads to an increment of implement steps of $(N-1) / N_{0}$

Table 3 : Parameters in CPU cost correlation

\begin{tabular}{cccc}
\hline Case No & MC algorithm & $a$ & $b$ \\
1D Size-dependent & CNMC (AAS) & $9.312 \times 10^{-6}$ & 2.212 \\
& SCVMC (AAS) & $4.368 \times 10^{-6}$ & 2.243 \\
& SCVMC (AAS) & $5.51 \times 10^{-5}$ & 0.928 \\
2D Size-independent & SCVMC (DAA) & $5.63 \times 10^{-7}$ & 1.794 \\
& CNMC (AAS) & $1.478 \times 10^{-4}$ & 0.845 \\
\hline
\end{tabular}

Since $H^{2}$ has a correlation with a square root of average number per size bin $\left(M N_{0} / n\right)^{1 / 2}, M N_{0} / n$ can be used to represent the accuracy $\left(Q_{C}\right)$ of the MC results instead of $H^{2}$. It gives

$$
Q_{c}=\frac{M N_{0}}{n}
$$

If $M$ is replaced by $Q_{c}$, the CPU cost is obtained as 


$$
C\left(M, N_{0}\right)=a Q_{c} n N_{0}^{b-1}
$$

$C\left(M, N_{0}\right)$ is a monotonic increasing function at $b>1$, so the minimum CPU cost of MC simulation is achieved at $N_{0}=1, M=Q_{C} n$. Alternatively, $C\left(M, N_{0}\right)$ is a monotonic decreasing function at $0<b<1$, so the minimum CPU cost of $M C$ simulation is achieved at $N_{0}=Q_{C} n, M=1$. In other words, if the CPU cost index is greater than one, a cost optimal, quality controlled simulation strategy is for a large number of replicates ( $M$ large) with small numbers of initial particles ( $N_{0}$ small). If the cost index is greater than one, the optimal strategy is for a single replicate $(M=1)$ and a large number of initial particles $\left(N_{0}\right.$ large $)$

The example of computational time saving at the $b>1$ condition can be seen in Table 4. The comparison is based on the one dimensional size-dependent coalescence case solved by the CNMC approach, $b=2.212$ (Table 3 ). Under the same accuracy criterion ( $n=433$ and $M N_{0}=10,000$ ), the computational time of 173.86 s for multiple MC simulation replicates $\left(N_{0}=500, M=20\right)$ is far less than the computational time of $6319.76 \mathrm{~s}$ for a single $M C$ simulation $\left(N_{0}=10,000, M=1\right)$. The example of computational time saving at the $0<b<1$ condition can be seen in Table 4 . The comparison is based on one dimensional size-dependent coalescence case solved by the CNMC approach $b=0.845$ (Table 3). Under the same accuracy criterion $\left(n=70^{2}\right.$, $M N_{0}=100,000$ ), the computational time of $2.48 \mathrm{~s}$ for a single MC simulation $\left(N_{0}=100,000, M=1\right)$ is able to save $37.2 \%$ CPU cost than that (3.95s) of multiple MC simulation replicates $\left(N_{0}=5,000, M=20\right)$.

Table 4. CPU cost at different CPU index

\begin{tabular}{cccccc}
\hline Case No & MC algorithm & $b$ & $N_{0}$ & $M$ & $C(\mathrm{~s})$ \\
& & & 500 & 20 & 173.86 \\
1D Size-dependent & CNMC (AAS) & 2.212 & 10,000 & 1 & 6309.76 \\
& & & 5,000 & 20 & 3.95 \\
2D Size-independent & CNMC(AAS) & 0.845 & 100,000 & 1 & 2.48 \\
\hline
\end{tabular}

\section{Conclusions}

Accuracy and optimal sampling strategy in Monte Carlo simulation of Population Balance Equations have been investigated in this study. It is concluded that Squared 
Hellinger Distance, $H^{2}$, is a powerful tool to measure the accuracy of MC simulation, and is related to initial sample number $\left(N_{0}\right)$, number of replicates $(M)$ and Number of bin sizes, $(n)$. The asymptotic approximation of $H^{2}$ is derived as $(1 / 8)\left(M N_{0} / n\right)^{-1 / 2}$ when $\left(M N_{0} / n\right)^{1 / 2}$ is large. Although the actual value of $H^{2}$ is higher compared to the theoretical trend in the 1D PBE cases, simulate results for both 1D and 2D PBEs with coalescence approximately demonstrated that scaling. A power law relationship, $C=$ $a M N_{0}{ }^{b}$ is found to describe the correlation between CPU cost and $N_{0}$, and $M$. The CPU cost index, $b$, illustrates the weight of $N_{0}$ in CPU cost.

Finally, an optimal sampling strategy is given as

1) $n$ determines the resolution of MC prediction and must be chosen by the user trading off the increased resolution available from increased $n$, with decreased accuracy ie increased $H^{2}$.

2) $M \times N_{0}$ determines the accuracy of $M C$ prediction, and both the accuracy of $M C$ simulation and the CPU cost increase as $M N_{0}$ increases. If the CPU cost index $b>1$, the minimum CPU cost is achieved for small numbers of $N_{0}$, and large values of $M$. Alternatively, if the CPU index, $0<b<1$, the minimum CPU cost is achieved at $M=1$ and a large value of $N_{0}$.

In this study, an optimal sampling strategy is developed for MC solution of PBEs with coalescence only. However, the approach can be extended to PBEs in any form solved by an MC approach. Substantial savings in computational cost are possible, if an optimal strategy is adopted.

\section{Acknowledgment}

The authors are grateful to AstraZeneca for funding, and to Professor John Biggins, of the School of Mathematics and Statistics at the University of Sheffield for introducing us to Hellinger's distance and the series approximation to the moments of the Poisson distribution.

\section{References}

1. Hounslow MJ, Ryall RL, Marshall VR, A discretized population balance for nucleation, growth, and aggregation, AIChE J. 1988;34: 1821-1832

2. Kumar S, Ramkrishna D, On the solution of population balance equations by discretization-I. A fixed pivot technique, Chem Eng Sci. 1996; 51:1311-1332 
3. Celnik M, Patterson R, Kraft M, Wagner W, Coupling a stochastic soot population balance to gas-phase chemistry using operator splitting, Combust.Flame. 2007; 148:158-176

4. Smith $M$, Matsoukas $\mathrm{T}$, Constant-number Monte Carlo simulation of population balances, Chem Eng Sci. 1998;53:1777-1786

5. Zhao H, Maisels A, Matsoukas T, Zheng C, Analysis of four Monte Carlo methods for the solution of population balances in dispersed systems, Powder Tech. 2007;173: 3850

6. Liffman K, A direct simulation Monte-Carlo method for cluster coagulation, J. Comput. Phys. 1992;100:116-127

7. Maisels A, Kruis FE, Fissan H, Direct simulation Monte Carlo for simultaneous nucleation, coagulation, and surface growth in dispersed systems, Chem Eng Sci. 2004;59: 2231-2239

8. Lin $\mathrm{Y}$, Lee $\mathrm{K}$, Matsoukas $\mathrm{T}$, Solution of the population balance equation using constant-number Monte Carlo, Chem Eng Sci. 2002; 57:2241-2252

9. Press WH, Teukolsky SA, Vetterling WT, Flannery BP, Numerical Recipes in Fortran 90. The Art of Parallel Scientific Computing, 2nd Edition, 1996, ISBN 0-521-57439-0.

10. Gillespie DT, The stochastic coalescence model for cloud droplet growth, J. Atmos. Sci. 1972;29:1496-1510

11. Gillespie DT, An exact method for numerically simulating the stochastic coalescence process in a cloud, J. Atmos. Sci. 1975;32:1977-1989

12. Daniel TG , A general method for numerically simulating the stochastic time evolution of coupled chemical reactions, J.Comput. Phys. 1976; 22:403-434

13. Kruis FE, Maisels A, Fissan $H$, Direct simulation Monte Carlo method for particle coagulation and aggregation, AIChE J. 2000;46:1735-1742

14. Gooch JRP and Hounslow MJ, Monte Carlo simulation of size-enlargement mechanisms in crystallization, AlChE J. 1996;42:1864-1874

15. Lattuada, $\mathrm{M}$ and $\mathrm{Wu} \mathrm{H}, \mathrm{A}$ simple model for the structure of fractal aggregates, $J$. Colloid Interface Sci. 2003;268:106-120.

16. Rosner DE, Yu S, MC Simulation of Aerosol Aggregation and Simultaneous Spheroidization, AlChE J. 2001; 47:545-561

17. Efendiev $Y$ and Zachariah MR, Hybrid Monte Carlo Method for Simulation of TwoComponent Aerosol Coagulation and Phase Segregation, J. Colloid Interface Sci. 2002;249:30-43

18. Tandon P, Rosner DE, Monte Carlo Simulation of Particle Aggregation and 
Simultaneous Restructuring, J. Colloid and Interface Sci.1999: 213:273-286

19. Zhao $\mathrm{H}$ and Zheng $\mathrm{C}$, Monte Carlo solution of wet scavenging of aerosols by precipitation, Atmos. Environ. 2006; 40:1510-1525

20. Braumann A, Goodson MJ, Kraft M, Mort PR, Modelling and validation of granulation with heterogeneous binder disperssion and chemical reaction, Chem Eng Sci. 2007;62:4717-4728

21. Braumann A, Kraft M, Mort PR, Parameter estimation in a multidimensional granulation model, Powder Tech.2010;197:196-210

22. Braumann A, Kraft M, Wagner W, Numerical study of a stochastic particle algorithm solving a multidimensional population balance model for high shear granulation, $J$. Comput. Phys. 2010;229:7672-7691

23. Braumann A, Man PLW, Kraft M, Statistical approximation of the inverse problem in multivariate population balance modeling, Ind. Eng.Chem. Res. 2010;49:428-438

24. Braumann A, Man PLW, Kraft M, The inverse problem in granulation modeling-two different statistical appraoches, AICHE J.2011;57:3105-3121

25. Rosenboom JG, Antonyuk S, Heinrich S,Kraft M, Characterisation of lactose powder and granules for multivariate wet granulation modelling, Chem. Eng. Sci. 2015;123:395-405

26. Wolfowitz J, The minimum distance method, Ann. Math. Stat. 1957; 28:75-88

27. Yang $S$, Minimum hellinger distance estimation of parameter in the random censorship model, Ann. Stat. 1991;19:579-602

28. Basu A, Harris IR, Basu S, Tests of hypotheses in discrete models based on the penalized Hellinger distance, Stat. Probabil. Lett. 1996; 27:367-373.

29. Karunamuni RJ, Wu J, Title Minimum Hellinger distance estimation in a nonparametric mixture model, J.Stat. Plan. Inference. 2009;139: 1118-1133

30. Gelbard F, Seinfeld JH, Numerical solution of the dynamic equation for particulate systems, J. Comput. Phys. 1978;28:357.

31. Vale HM, McKenna TF, Solution of the population balance equation for twocomponent aggregation by an extended fixed pivot technique, Ind. Eng.Chem. Res. 2005; $44: 7885-7891$

32. Kendall S, Stuart MA, Ord JK, The advanced theory of statistics. High Wycombe: Charles Griffin. 1983

33. Hounslow MJ, A discretized population balance for continuous systems at steady state, AIChE J. 1990;36:106-116 


\section{List of Figures}

Fig 1. Flowchart of MC solution of coalescence PBEs

Fig 2. Comparison between particle mass distribution (at constant size bins $n=21$ ) obtained by CNMC $\left(P_{i}=P\left(m_{i} \leq m<m_{i+1}\right), \bar{m}_{i}=\left(m_{i+1}+m_{i}\right) / 2\right)$ and analytical solution $\left(P_{i}\left(\bar{m}_{i}\right)=\int_{m_{i}}^{m_{i+1}} n(m) d m\right)$ for $1 \mathrm{D}$ case (size-dependent coalescence) (a) at constant $M=1$ (b) at constant $M=20$ (c) at constant $M N_{0}=10,000$

Fig 3.Calculated (CNMC and SCVMC at size bins $n=13-13,000)$ and asymptotic approximation $H^{2}$ (when $\left(M N_{0} / n\right)^{1 / 2}$ is large) dependence of $\left(M N_{0} / n\right)^{1 / 2}$ for $1 \mathrm{D}$ case (size-dependent coalescence) at constant $M=20$

Fig 4. Calculated (CNMC and SCVMC at size bins $n=13-13,000)$ and asymptotic approximation $1-H^{2}$ (when $\left(M N_{0} / n\right)^{1 / 2}$ is small) dependence of $\left(M N_{0} / n\right)^{1 / 2}$ for $1 \mathrm{D}$ case (size-dependent coalescence) at constant $M=20$

Fig 5. 3D plot of particle mass $\left(m_{1}, m_{2}\right)$ distribution of analytical solution for 2D case (sizeindependent coalescence) (a) at $\tau=0\left(n_{0, \max }=0.108\right)$ (b) at $\tau=100\left(n_{\tau, \max }=4.29 \times 10^{-5}\right)$

Fig 6.Calculated (CNMC and SCVMC at size bins $n=7^{2}-350^{2}$ ) and asymptotic approximation $H^{2}$ (when $\left(M N_{0} / n\right)^{1 / 2}$ is large) dependence of $\left(M N_{0} / n\right)^{1 / 2}$ for 2D case (size-independent coalescence) at constant $M=20$

Fig 7. Averaged (constant $M=20) \mathrm{CPU}$ cost dependence of $N_{0}$ for $1 \mathrm{D}$ case (size-dependent coalescence) using methods (CNMC, SCVMC) and 2D case (size-independent coalescence) using methods (CNMC, SCVMC (AAS and DAA)), the CPU cost index $(b)$ of each MC algorithm is in Table 3

\section{List of Tables}

Table 1: Summary of application of Monte Carlo method to PBEs in the literatures

Table 2: Parameters used in MC simulation

Table 3 : Parameters in CPU cost correlation

Table 4. CPU cost at different CPU index 